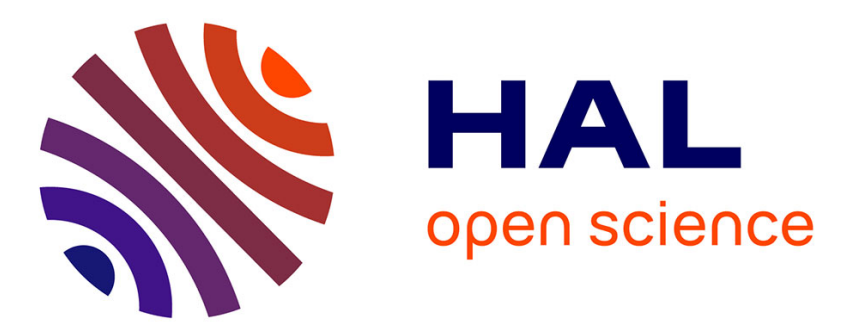

\title{
A framework for closed-loop flow control using the parabolized stability equations
}

Kenzo Sasaki, André V. Cavalieri, Flavio J. Silvestre, Peter Jordan, Gilles Tissot, Damien Biau

\section{- To cite this version:}

Kenzo Sasaki, André V. Cavalieri, Flavio J. Silvestre, Peter Jordan, Gilles Tissot, et al.. A framework for closed-loop flow control using the parabolized stability equations. 23rd AIAA/CEAS Aeroacoustics Conference, 2017, Denver, United States. pp.1-11, 10.2514/6.2017-3003 . hal-02443472

\section{HAL Id: hal-02443472 \\ https://hal.science/hal-02443472}

Submitted on 17 Jan 2020

HAL is a multi-disciplinary open access archive for the deposit and dissemination of scientific research documents, whether they are published or not. The documents may come from teaching and research institutions in France or abroad, or from public or private research centers.
L'archive ouverte pluridisciplinaire HAL, est destinée au dépôt et à la diffusion de documents scientifiques de niveau recherche, publiés ou non, émanant des établissements d'enseignement et de recherche français ou étrangers, des laboratoires publics ou privés. 


\title{
A framework for closed-loop flow control using the parabolized stability equations
}

\author{
Kenzo Sasaki;, André V. G. Cavalieri†, \\ Flávio J. Silvestre \\ Gilles Tissot ${ }^{\top}$ and Damien Biau!
}

\begin{abstract}
We develop a reduced-order-model framework using the parabolized stability equations and identification techniques for the closed-loop control of unsteady fluctuations along fluidic systems. These models had been successfully applied to a turbulent jet as estimation techniques and to an incompressible shear-layer for the development of closed-loop control laws. Through this paper, we propose a further investigation of the PSE-based transfer functions, exploring its flexibility to educe different control schemes and to determine the most effective sensor/actuator positions. Emphasis is be given to the feedforward and feedback configurations for flow control, and differences are understood in terms of causality. A study of the robustness to uncertainties in Reynolds and mean flow velocity, along with external perturbations is also presented. These topics allow deeper insight into the active closed-loop flow control problem and therefore may lead to more effective schemes, particularly on what concerns the experimental implementation of closed-loop control.
\end{abstract}

\section{Introduction}

Altering the behaviour of a flow, either passively or actively, in open or closed-loop may lead to several industrial and academic applications, which are related to the modification of the growth of the unsteady fluctuations along the flow system.

Applications are related both to the increase or attenuation of the growth of fluctuations. An augmentation of combustion mixing efficiencies ${ }^{1}$ is an example of the first, whereas a decrease in the drag via laminar-turbulent transition delay ${ }^{2,3}$ is an application of the second.

In spite of the several foreseeable uses of flow control, there are major obstacles that need to be overcome; ${ }^{4}$ The inherent non-linearity of the Navier-Stokes equations and the large dimension of a fluidic problem, as each discretized point of a grid may be regarded as a state variable to be controlled or observed. These two characteristics make it difficult to propose a model that supplies the appropriate balance between computational requirements and accuracy of the prediction. Furthermore, based on a limited set of measurements (inputs) an actuation must be imposed in the system in order to alter the amplitude of the instability waves at fixed positions (outputs). Determination of the most sensitive positions for actuation and sensing is also of considerable relevance, overcoming this issue may allow for more efficient control schemes.

A promising idea to tackle these issues has been the use of reduced-order models to the estimation of fluctuations and the effect of an actuation into the flow. ${ }^{5,6}$ These models are then used to the derivation of linear control laws and tested a posteriori in an experiment or non-linear simulation. The complication now becomes how to obtain a reduced-order model that is both computationally inexpensive and accurate in capturing the said relationship between inputs and outputs of the system.

*PhD Student, Instituto Tecnológico de Aeronáutica,São José dos Campos, Brazil.

${ }^{\dagger}$ Professor, Instituto Tecnológico de Aeronáutica,São José dos Campos, Brazil.

¥Professor, Instituto Tecnológico de Aeronáutica,São José dos Campos, Brazil.

$\S$ Research Scientist, Institut Pprime, CNRS- University of Poitiers-ENSMA, Poitiers, France.

『 Post-doc Student, Institut de Mathmatiques de Toulouse, Universit Paul Sabatier, Toulouse, France.

" École Nationale Supérieure d'arts et Métiers, Paris, France. 
In previous studies by this group we introduced the PSE-based transfer function which presented a compelling result in predicting the open-loop behaviour of the unsteady fluctuations along a jet ${ }^{7}$ and through which closed-loop control was obtained for a shear-layer. ${ }^{4}$ The objective of this work is to further explore PSE-based closed-loop control using the two-dimensional shear layer as a prototypical problem, for which different control schemes may be tested. Constraints such as uncertainties and external perturbations may be reproduced in the simulation, to determine the effectiveness of the control. The flexibility of the PSE transfer function which allows the prediction between any positions, with different levels of effectiveness, is very much desired and will be explored during the determination of the sensor/actuator pair.

This paper is organized as follows; in section II previous results concerning the estimation and actuation problems are recalled, section III presents the feedback and feedforward control problems. The methodology for determination of the sensor and actuator positions is presented in sectio IV. Results concerning closedloop control along with a robustness evaluation is presented in sections V and VI. Finally, conclusions are drawn in section VII.

\section{Previous results}

Through this section, some of the previously obtained results, which will be necessary in the remaining of the paper will be recalled. The data is divided into the estimation and actuation problems, respectively. The emphasis will be over the two-dimensional shear layer, over which the closed-loop control is applied. For results concerning the application of time-domain estimations for a turbulent jet, the reader is referred to. ${ }^{8}$

\section{A. Estimation problem}

We briefly revise the estimation results applied for the shear-layer, this simulation is the same used in the study in Biau, 2016. ${ }^{9}$ This flow corresponds to a two-dimensional incompressible problem, of Reynolds number $R e=100$. For further information regarding this system, the reader is referred to Sasaki et al., $2016{ }^{4}$ The strategy to be followed consists in application of the PSE transfer function, however analogous results may be obtained using the empirical transfer functions based in the cross and auto power spectral densities, with a much higher computational effort. For a careful derivation of these methodologies, the reader is referred to Sasaki et al., 2016. ${ }^{4}$

Figure 1 presents the estimation results for a single-input-multiple-outputs prediction using the PSE transfer function, in comparison with the direct-numerical-simulation results. Amplitudes and phases are appropriately captured, particularly if input and output positions are close to each other. Results considering single-input-single-output models also show compelling results, however they will not be shown for the sake of brevity of this paper.

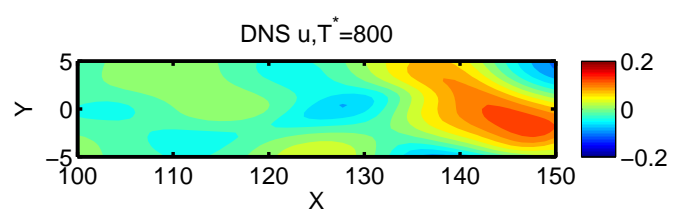

(a)

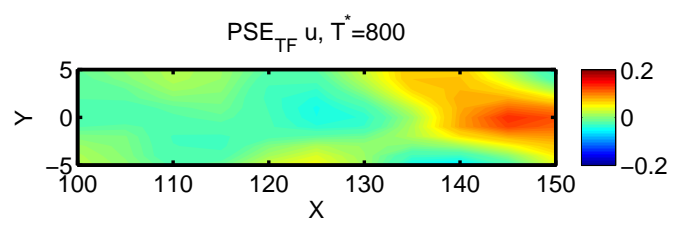

(c)

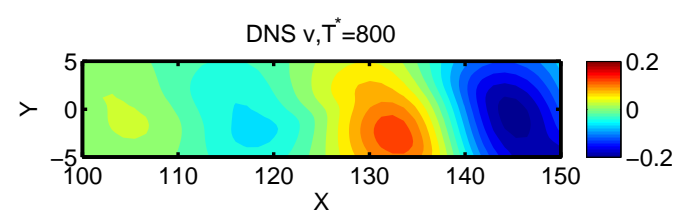

(b)

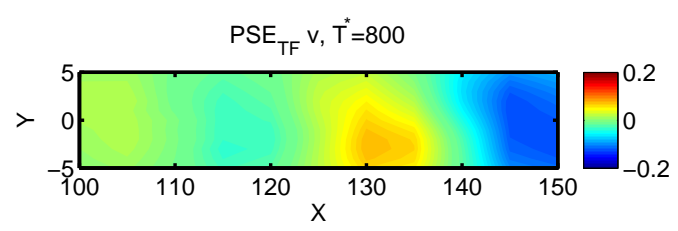

(d)

Figure 1. DNS of the bidimensional shear-layer, in (a) and (b), in comparison against PSE transfer functions prediction from a single measurement, in (c) and (d). 


\section{B. Actuation problem}

The second necessary ingredient for active control is an actuation. For the shear-layer, an actuation may be considered as a body-force, which seeks to model aa actuator into the flow. Equation 1 determines the force to be applied to the system,

$$
f(x, y, t)=u(t) e^{-\frac{-\left(x-x_{2}\right)^{2}}{L^{2}}} e^{\frac{-\left(y-y_{2}\right)^{2}}{L^{2}}}
$$

where $\left(x_{2}, y_{2}\right)$ represent the position where the actuation is installed and $L$ is its spatial support, $a(t)$ is a broadband time signal, expected to be able to act along the unstable frequencies of the problem. For a review into the actuator types, the reader is referred to Cattafesta and Sheplak, 2011. ${ }^{10}$

There are now two strategies to determine the effect of $f(x, y, t)$ into the flow; The empirical transfer function considering the auto and cross spectrum densities between $a(t)$ and a chosen output position may be taken; Or this body force may be projected into the Kelvin-Helmholtz mode, to determine how it will alter the flow locally, PSE may then be used to account for its effect downstream. The first one is based on the System's theory of frequency responses, ${ }^{11}$ whereas the second one comes from the signalling problem defined in the stability theory of flows. ${ }^{12-14}$ Results of both of these strategies may be found in Sasaki et $a l ., 2016,{ }^{4}$ and will not be presented here, but have been found to be equivalent and to reproduce the actual non-linear simulation results with reasonable accuracy.

\section{Feedback and feedforward control strategies for flow control}

One of the most important classifications when dealing with active flow control regards the separation between feedback and feedforward control strategies. Although hardly ever used alone i.e. without a feedback loop, for system's applications, feedforward has seen several uses in flow control, particularly in convectively unstable flows, such as the one that will be dealt with in this section.

The bibliography on the subject is vast, dealing with both strategies with a flow control mind set. To feedback control, the reader is referred to the works of Dadfar et al., 2015, Bagheri et al., 2009, Semeraro et al., 2013 and 2011 ${ }^{5,15-18}$ all of which deal with different control-laws for the reduction of Tollmien-Schlichting waves in boundary layers, both in bi-dimensional and three-dimensional cases. For the feedforward control, $\mathrm{Li}$ and Gaster, 2006 and Fabbiane et al., 2015 ${ }^{2,19}$ use wave cancellation techniques to control boundarylayer instabilities and Gautier and Aider, $2014^{20}$ use an ARMAX-based control for a backward-facing step problem. The works of Belson et al., 2013 and Fabbiane et al., 2014 ${ }^{3,6}$ provide insight into the differences between these two strategies applied to flow control.

The main difference between feedforward and feedback, in a flow with convective nature, is related to whether or not the actuator will affect the input position; For the first case, the actuator does not affect in the measurement of the input such that it acts by opposite phase cancellation of the output. If the actuator affects the input signal, the resulting scheme is denoted as feedback.

For strongly convective flows, a feedforward scheme will result if the actuator is downstream of the input and feedback if the input is downstream of the actuation.

For these flows, feedback only leads to significant performances if the positions of the sensor and actuator are close to one another, ${ }^{3,6}$ otherwise the actuation will be unable to alter the flow accordingly. An evaluation of the Bode diagram of the closed-loop control and the corresponding phase margins, along with the sensitivity transfer functions, will supply the necessary information on whether or not the chosen position is appropriate. Care must be taken as the feedback control will change the position of the poles of the closed-loop system, and it is therefore capable of de-stabilizing the plant; feedforward, on the other hand, is unable to do so.

Nevertheless, the main disadvantage of feedforward control is that it is unable to deal with perturbations downstream of the sensor position making its use in practical applications more challenging. Differences between model and the real system are also not seen on a purely feedforward loop, and although it will not make the plant unstable, its effectiveness may be reduced rapidly in such cases. Such differences, when known a priori may be quantified and will allow for the definition on whether or not feedforward alone will work. ${ }^{21}$

Given that each of these strategies presents different strengths and drawbacks, one of the main objectives of this work is to design feedback and feedforward control laws and determine the differences in the performance of these.

Through the remaining of this paper, the following nomenclature will be considered; $y(t), u(t)$ and $z(t)$ will denote the time behaviour of input, actuation and output signals, $G_{i j}$ represents a transfer function 
between positions at $i$ and $j$.

Figures 2 and 3 present usual block diagrams applied for feedback and feedforward control strategies, and are those that will be considered in this work.

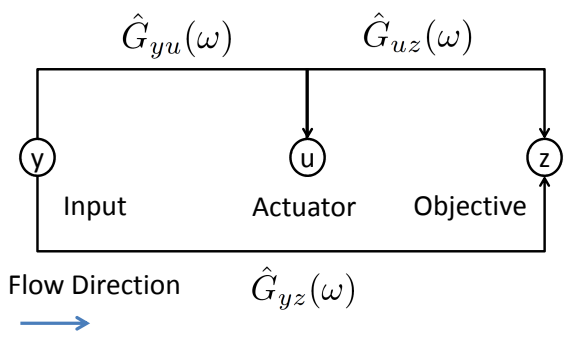

(a)

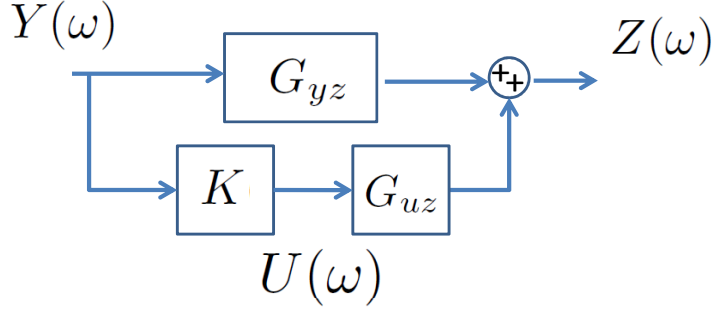

(b)

Figure 2. Feedforward scheme for control in (a) and resulting block diagram for this strategy in (b).

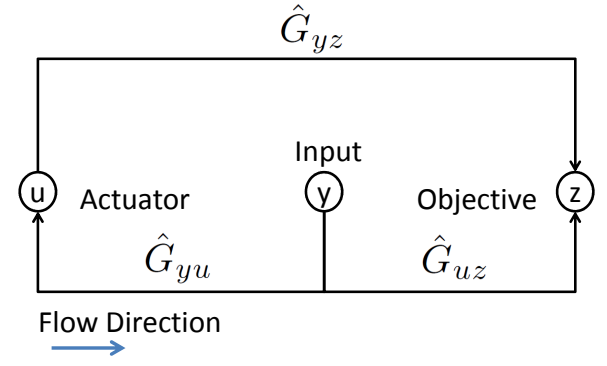

(a)

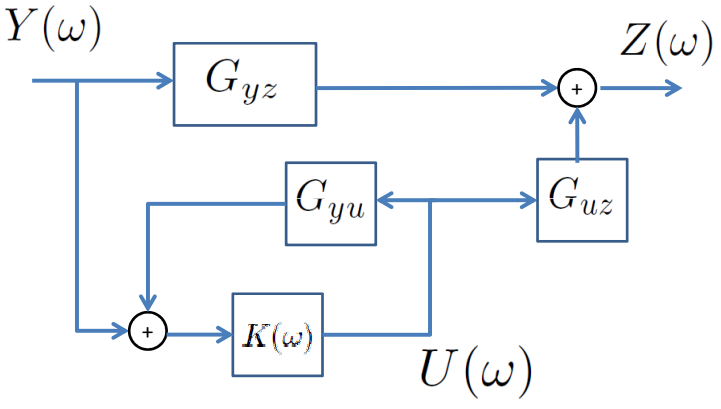

(b)

Figure 3. Feedback scheme for flow control in (a) and resulting block diagram for this strategy in (b).

The previous block diagrams do not consider the presence of exogenous disturbances, which could be added anywhere on the actuator-sensor-objective branch. The resulting closed-loop relations between input and output signals are shown in equations 2 and 3.

$$
\begin{aligned}
& Z(\omega)=\left[G_{y z}(\omega)+K G_{u z}(\omega)\right] Y(\omega)(\text { Feedforward }) \\
& Z(\omega)=\left[G_{u z}(\omega)+\frac{K G_{u z}(\omega)}{1-K G_{u y}(\omega)}\right] Y(\omega)(\text { Feedback })
\end{aligned}
$$

With the block diagrams assembled, the control strategy consists into minimizing the transfer function between input and output positions, such that the fluctuations at the position of the objective are attenuated. We choose to perform an exact inversion i.e., solving equations 2 and 3 for $K(\omega)$ such that the output is exactly zero, this method is defined as wave-cancellation. The actuation will then be calculated via a convolution of the input with the gain, as per equation 4.

$$
u(t)=-\int_{-\infty}^{\infty} k(\tau) y(t-\tau) \mathrm{d} \tau,
$$




\section{Choosing the control positions}

Prior to the application of closed-loop control for the velocity fluctuations along the shear-layer, a determination of the most suitable sensor and actuator positions shall be performed. This will be done by considering three aspects; sensitivity to forcing, causality and accuracy of the reduced-order-models in reproducing the resulting behaviour from a non-linear simulation.

To determine the most sensitive areas to forcing, we project a body force in the shape of a Dirac delta in the Kelvin-Helmholtz mode,

$$
f(X, Y)=\delta\left(Y-Y_{2}\right) \delta\left(X-X_{2}\right)
$$

such that

$$
\hat{\hat{f}}=\delta\left(Y-Y_{2}\right) \mathrm{e}^{-\mathrm{i} \alpha X_{2}},
$$

given the definition of the Fourier transform and the filtering property of the delta. For this case, the amplitude of the projection onto the Kelvin-Helmholtz mode is given exclusively by the adjoint mode at $Y=Y_{2}$, with an exponential-like behaviour along the axial direction, as per equation 7. Further details concerning the determination of the effect of a body force into the flow may be found in. ${ }^{4}$

$$
\left\langle\hat{\hat{\Psi}}_{K H}(\alpha, Y, \omega), \hat{\hat{f}}(\alpha, Y, \omega)\right\rangle=\hat{\hat{\Psi}}_{K H}(\alpha, Y, \omega) e^{-i \alpha X_{2}} .
$$

This corresponds to finding the $\delta\left(Y-Y_{2}\right) \delta\left(X-X_{2}\right)$ response of the system. Thus it may be used to determine sensitivity characteristics of the flow, leading to the optimal positioning of actuators along transverse directions. Defining the parameter $\kappa_{u}$ and $\kappa_{v}$,

$$
\begin{aligned}
\kappa_{u} & =\int_{-\infty}^{\infty}\left|\hat{\hat{\Psi}}_{u}(\alpha, Y, \omega)\right|^{2} d \omega \\
\kappa_{v} & =\int_{-\infty}^{\infty}\left|\hat{\hat{\Psi}}_{v}(\alpha, Y, \omega)\right|^{2} d \omega
\end{aligned}
$$

where $\hat{\hat{\Psi}}_{u}(\alpha, Y, \omega)$ and $\hat{\hat{\Psi}}_{v}(\alpha, Y, \omega)$ are the values of the adjoint mode at $Y_{2}$ corresponding to axial and transverse forcings, respectively. The greater the value of these parameters, the more efficient will be body force, introduced at $Y_{2}$, in producing a response in the controllable frequencies of the problem. Figure 4 presents the value of these sensitivity indexes, at the position $X_{2}=100$, indicating that it is ideal to act along the centerline of the flow, in order to obtain the highest response.

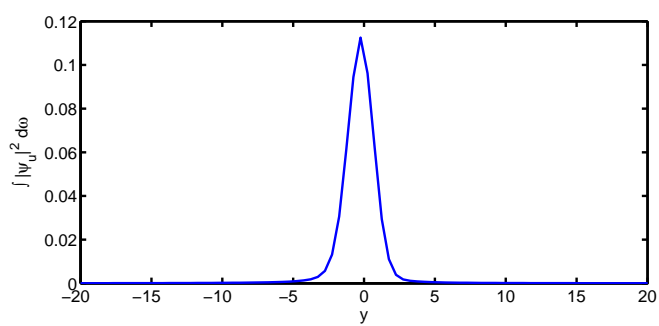

(a)

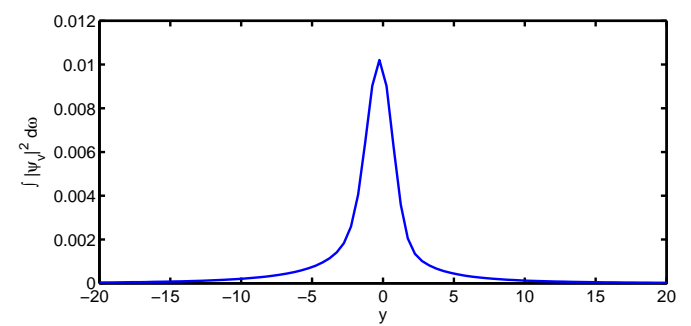

(b)

Figure 4. Sensitivity index for: (a) streamwise forcing along $u$ and (b) transverse forcing along $v$.

It should be noted that given the exponential behaviour of the Kelvin-Helmholtz mode, the parameter $\kappa$ will always be higher at upstream positions. This fact is not necessarily representative of the actual behaviour observed in the non-linear simulation and therefore other metrics are necessary to define the streamwise position for the actuation.

We start by determining the causality of the resulting gain in closed-loop. Consider equation 4, this convolution can only be taken, in practice, from $t=0$ to $t=\tau$ as information for negative values of time will violate the causality of the system. 
A suitable choice of positions for input, actuator and output should then result in a causal gain, to maximize its effect over the actuation. Such information is normally available a posteriori, as in, ${ }^{6}$ where the gain is evaluated for several positions and analysis of the amplitude of the gain for negative values of $t$ would allow a quantification of causality. An alternative way to perform this task is to use the Hilbert transform, ${ }^{22}$ which has the added advantage of testing combinations in a fast, computationally efficient manner, without the need to perform several non-linear simulations.

If the imaginary part of the frequency response (or in this case, a transfer function) of a linear system is equal to the Hilbert transform of the real part, such system represents a causal, linear filter, and knowledge of the real part is sufficient to completely specify the system, the imaginary part adding redundant information. Therefore, a check for causality is to compare the imaginary part of the transfer function with the Hilbert transform of the real part $\left(\mathrm{see}^{23}\right)$.

One way to evaluate this in a quantitative way is to compute the correlation between the Hilbert transform of the real part of the transfer function, $\hat{H}\left(\Re\left(G_{I 13}\right)\right)$ and the imaginary part of $G_{I 13}$. This parameter is given by

$$
P=\max \left(\frac{\int_{-\infty}^{\infty} \hat{H}(\Re(G(\omega))) \Im(G(\omega)) d \omega}{\sqrt{\int_{-\infty}^{\infty} \hat{H}(\Re(G(\omega)))^{2} d \omega} \sqrt{\int_{-\infty}^{\infty} \operatorname{Im}(G(\omega))^{2} d \omega}}\right) .
$$

High values of $P$ will indicate a causal transfer function, which will therefore lead to a causal gain.

Figure 5 presents a plot of the parameter $P$, considering the input at $\left(x_{0}, y_{0}\right)=(75,0)$, dark areas indicate non-causal behaviour of the transfer function.

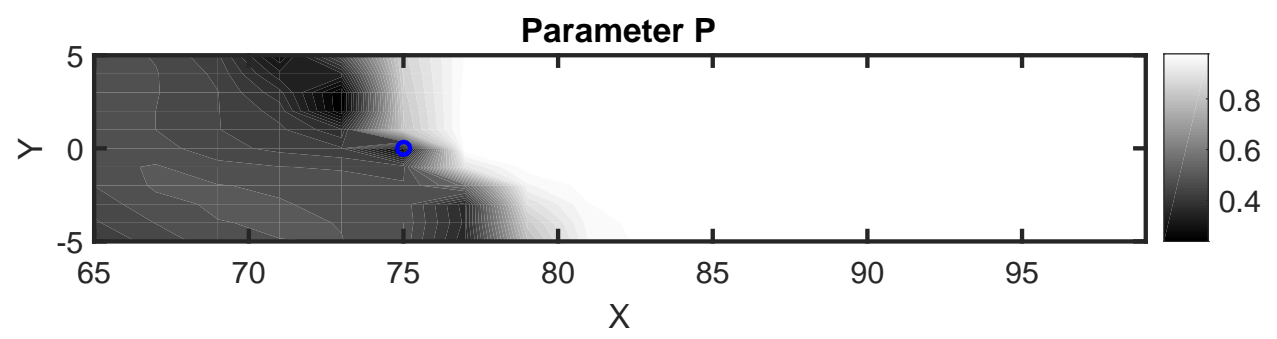

Figure 5. $P$ parameter for evaluation of the causality of the transfer function. Input is in the circle at $\left(x_{0}, y_{0}\right)=(75,0)$.

It is noticeable that if the output is downstream of the input, this results in low correlations, indicating a highly non-causal transfer function. If the output is downstream, but within one wavelength of the most unstable frequency of distance the correlations are also low, determining a minimal separation distance between sensors.

The third metric used to choose suitable positions for closed-loop implementation is the normalized peak correlation between the estimation and non-linear simulation, $\operatorname{Corr}=\max \left(C_{z_{1} z}(\tau)\right)$, where $C_{z_{1} z}(\tau)$ is defined as

$$
C_{z_{1} z}(\tau)=\frac{\int_{-\infty}^{\infty} z_{1}(t) z(t+\tau) d t}{\sqrt{\int_{-\infty}^{\infty} z_{1}^{2}(t) d t} \sqrt{\int_{-\infty}^{\infty} z^{2}(t) d t}},
$$

where $z_{1}$ and $z$ are the real and estimated signals, respectively.

The control signal is expected to be more effective in regions of high values of this parameter, which indicate the linear model gives a good estimation of the non-linear dynamics.

Figure 6 presents the peak correlation for both estimation and actuation transfer functions, at four different input/output pairs, separated by $\Delta X=25$.

For both estimation and actuation transfer functions, we observe that predictions lose accuracy with increasing distance, and that the use of downstream inputs also leads to a decrease of correlations between predictions and DNS results. Both trends can be attributed to non-linearity, as the evolution of the mixing layer leads to vortex roll-up and pairing which can only be only approximately accounted for by the linearised model. ${ }^{24}$ The chosen set of positions for closed-loop control were then $X=75,100$ and 125, for input, 


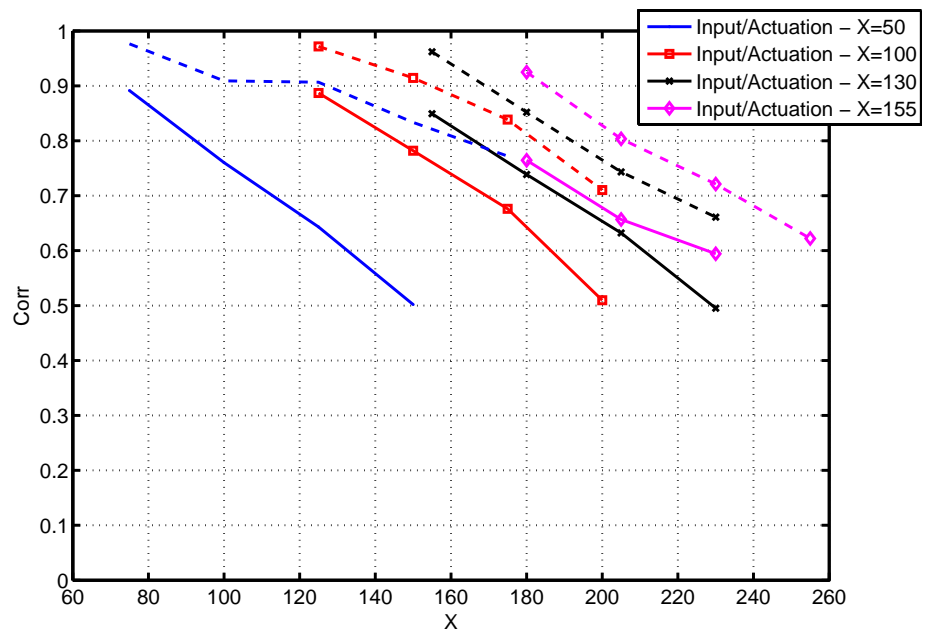

Figure 6. Peak correlation between prediction and DNS for the estimation and actuation transfer functions. Solid and dashed lines represent the correlations obtained for the actuation and estimation transfer functions, respectively.

actuation and output, respectively, in a feedforward scheme, all of which taken in the centerline of the flow, where the sensitivity to external forcing is highest.

For the feedback scheme, several combinations were evaluated, in the centerline of the flow, however, only when the separation distance was below $\Delta X=2$ noticeable reductions of the output fluctuations were present.

\section{Results}

Figure 7 presents the transverse velocity fluctuations for the feedforward and feedback strategies, acting via a transverse forcing. Given the strong convective nature of this flow, leading to non-causal gains rapidly as the actuation is positioned upstream of the input, the feedback scheme presents a much inferior performance to feedforward.
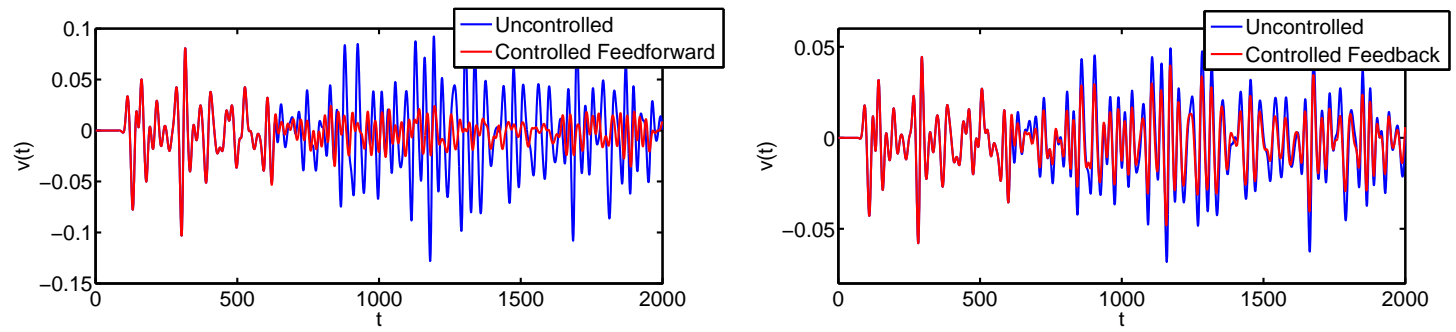

Figure 7. Transverse velocity attenuations obtained from feedforward and feedback strategies, input, actuation and output were located at $(x, y)=(75,0),(100,0)$ and $(125,0)$ and $(x, y)=(102,0),(100,0)$ and $(104,0)$, for feedforward and feedback, respectively.

The resulting turbulent kinetic energy, for the feedfoward control scheme, using either a transverse or axial forcing compared against the uncontrolled case is shown in figure 8. A reduction of up to $50 \%$ is observed downstream of actuation.

Finally, vorticity snapshots of the uncontrolled and controlled cases are presented in figure 9 . As the control action takes place, vortex roll-up and pairing is delayed. This supplies evidence that for the analogous compressible mixing layer problem ${ }^{24-26}$ or low Reynolds number jet ${ }^{27,28}$ the control action would result in lower sound radiation. 


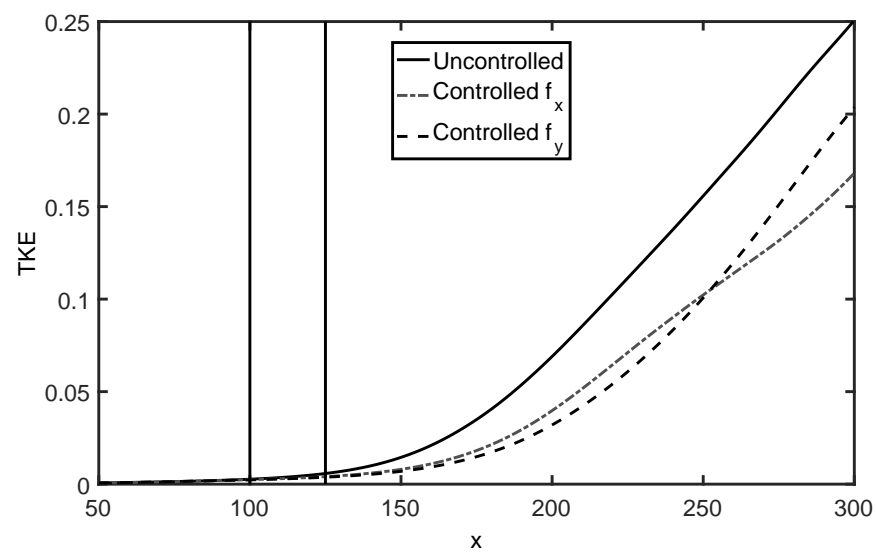

Figure 8. Turbulent kinetic energy for the uncontrolled case and wave cancellation via $f_{x}$ and $f_{y}$, vertical lines indicate the axial position of input and objective, respectively.

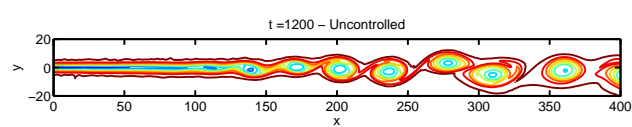

(a)

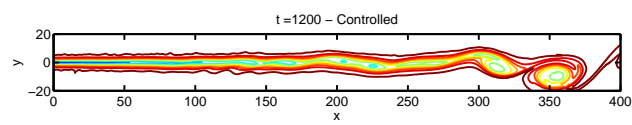

(c)

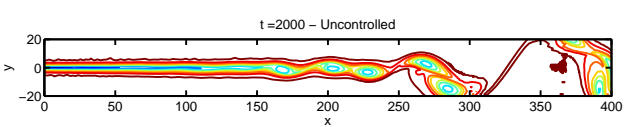

(b)

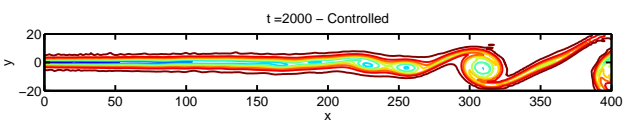

(d)

Figure 9. Comparison of the vorticity fluctuations for the uncontrolled (a and b) and controlled (c and d) cases. The delay in the vortex pairing becomes apparent. 


\section{Robustness evaluation}

The robustness of the control law was evaluated considering Reynolds, mean flow velocity, amplitude of the inflow perturbations and perturbations inserted between input and output positions. Given the low effectiveness of the feedback scheme for this flow, only the feedforward law was evaluated.

Figure 10 presents the quotient between the root-mean-square signals of the controlled and uncontrolled cases for the axial and transverse velocity fluctuations. As in the previous sections, the objective of the controller was to attenuate the transverse velocity fluctuations.

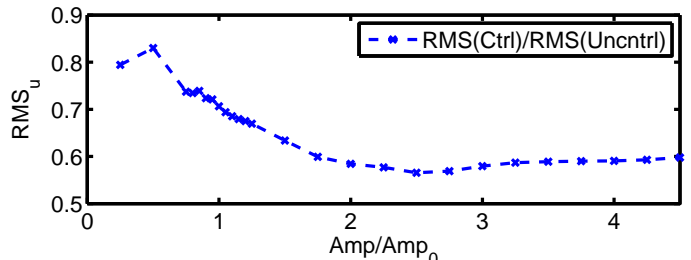

(a)

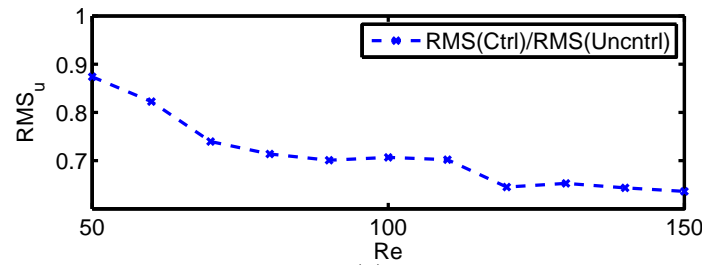

(c)

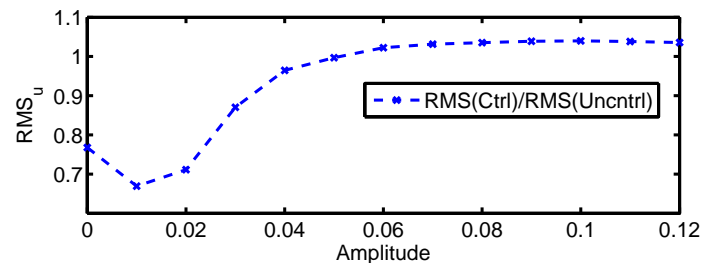

(e)

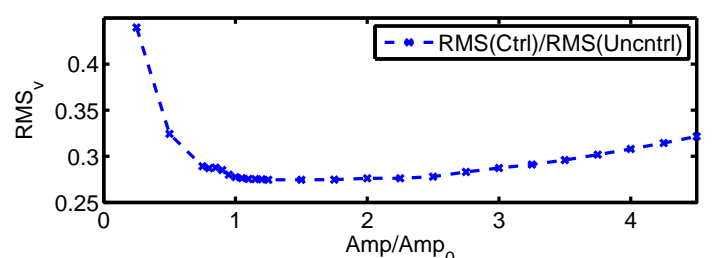

(b)

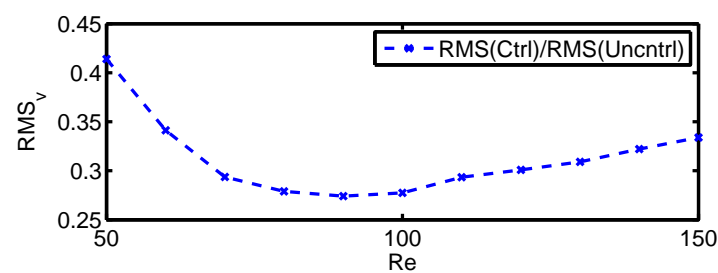

(d)

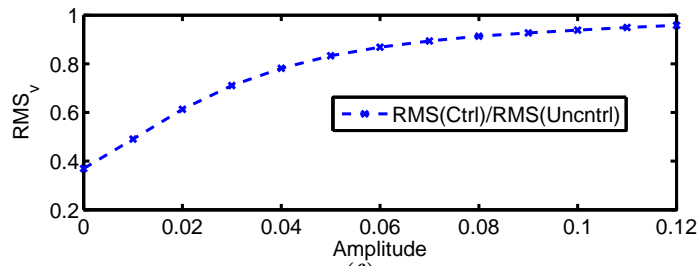

(f)

Figure 10. Robustness verification for perturbations inserted between input and objective. RMS of the streamwise and transverse velocity components of the controlled case divided by the corresponding openloop scenario. The same combination of positions and objective as that of figure 7 were considered; (a), (b) amplitude of the inflow perturbations, (c), (d) Reynolds number and (e), (f) perturbations in the form of a broadband forcing were evaluated.

It is observed that the system is fairly insensitive to the amplitude of the inflow perturbation and Reynolds number, particularly due to the fact that the linear approximation remains valid for these higher amplitudes.

The greatest drawback of the feedforward control scheme is observed when there are perturbations present between input and output positions. For this case, even for low amplitude of the perturbations the result for the closed-loop system rapidly degrades up to the the point when the control law is completely ineffective.

\section{Conclusions}

We have developed a framework for the closed-loop control of fluctuations, which was based in frequencydomain transfer functions, obtained either via PSE or empirical frequency responses. These reduced-ordermodels allow direct derivation of feedback and feedforward laws for the closed-loop control of the fluctuations.

The PSE transfer functions allowed further insight into the physics of the flow, permitting an initial estimation of the most effective positions for sensor and actuator placement, which was based in three metrics; causality, sensitivity to forcing and accuracy of the reduced-order models in reproducing the behaviour of the non-linear simulation. This is made without the need to compute and test the control law in the DNS for each case, leading to a significant computational gain.

Application was made to an unstable shear-flow, the highly convective nature of this flow caused the 
feedback scheme to be much less effective than the feedforward, differences being understood in terms of the causality of the resulting transfer functions between sensors and actuator. For the chosen set of positions, the feedforward control-law led to reductions of the order of $50 \%$ in the turbulent kinetic energy, accompanied by a delay in the vortex pairing and roll-up, a desirable effect in aeroacoustic applications.

Experimental implementation of these control laws for a turbulent jet is yet to be demonstrated. Other than the more complex dynamics of such flow, experimental constraints, measurement noise and model uncertainties constitute a more challenging task for the controller. The use of the DNS allowed for the replication of some of these constraints and showed that, for the case of the bi-dimensional shear layer, the greatest concern is related to unsensed perturbations, present downstream of the input sensors, with the feedforward scheme demonstrating an adequate performance to model uncertainties, such as Reynolds and mean-flow velocity differences. Therefore, the numerically implemented control laws presented in this paper are a useful tool for the proof of concepts, constituting an important step towards the further goal of controlling a turbulent jet in closed-loop.

\section{Acknowledgements}

The authors acknowledge funding from CNPq (grant number 444796/2014-2). Kenzo Sasaki acknowledges the financial support from Capes via a PhD scholarship. André V. G. Cavalieri was supported by $\mathrm{CNPq}$ through a research scholarship.

\section{References} 417 .

${ }^{1}$ Kim, J. and Bewley, T. R., "A linear systems approach to flow control," Annu. Rev. Fluid Mech., Vol. 39, 2007, pp. 383-

${ }^{2}$ Fabbiane, N., Simon, B., Fischer, F., Grundmann, S., Bagheri, S., and Henningson, D. S., "On the role of adaptivity for robust laminar flow control," Journal of Fluid Mechanics, Vol. 767, 2015, pp. R1.

${ }^{3}$ Fabbiane, N., Semeraro, O., Bagheri, S., and Henningson, D. S., "Adaptive and model-based control theory applied to convectively unstable flows," Applied Mechanics Reviews, Vol. 66, No. 6, 2014, pp. 060801.

${ }^{4}$ Sasaki, K., Tissot, G., Cavalieri, Andr V. G.and Jordan, P., and Biau, D., "Real-time control of wavepacket in a free shear-layer," 22nd AIAA/CEAS Aeroacoustic Conference and Exhibit, 2016.

${ }^{5}$ Semeraro, O., Bagheri, S., Brandt, L., and Henningson, D. S., "Feedback control of three-dimensional optimal disturbances using reduced-order models," Journal of Fluid Mechanics, Vol. 677, 2011, pp. 63-102.

${ }^{6}$ Belson, B. A., Semeraro, O., Rowley, C. W., and Henningson, D. S., "Feedback control of instabilities in the twodimensional Blasius boundary layer: the role of sensors and actuators," Physics of Fluids (1994-present), Vol. 25, No. 5, 2013, pp. 054106 .

${ }^{7}$ Sasaki, K., Piantanida, S., Cavalieri, A. V., and Jordan, P., "Real-time modelling of wavepackets in turbulent jets," 21 th AIAA/CEAS Aeroacoustic Conference and Exhibit, 2015.

${ }^{8}$ Sasaki, K., Piantanida, S., Cavalieri, V. G., and Jordan, P., "Real-time modelling of wavepackets in turbulent jets," Manuscript in press for publication.

${ }^{9}$ Biau, D., "Transient growth of perturbations in Stokes oscillatory flows," Journal of Fluid Mechanics, Vol. 794, 2016, pp. R4.

${ }^{10}$ Cattafesta, L. N. and Sheplak, M., "Actuators for active flow control," Annual Review of Fluid Mechanics, Vol. 43, 2011, pp. 247-272.

${ }^{11}$ Bendat, J. S. and Piersol, A. G., Random data: analysis and measurement procedures, Vol. 729, John Wiley \& Sons, 2011.

${ }^{12}$ Huerre, P. and Monkewitz, P. A., "Local and global instabilities in spatially developing flows," Annual review of fluid mechanics, Vol. 22, No. 1, 1990, pp. 473-537.

${ }^{13}$ Huerre, P. and Monkewitz, P. A., "Absolute and convective instabilities in free shear layers," Journal of Fluid Mechanics, Vol. 159, 1985, pp. 151-168.

${ }^{14}$ Huerre, P., Batchelor, G., Moffatt, H., and Worster, M., "Open shear flow instabilities," Perspectives in fluid dynamics, 2000, pp. 159-229.

${ }^{15}$ Dadfar, R., Hanifi, A., and Henningson, D. S., "Feedback Control for Laminarization of flow over Wings," Flow, Turbulence and Combustion, Vol. 94, No. 1, 2015, pp. 43-62.

${ }^{16}$ Bagheri, S., Brandt, L., and Henningson, D. S., "Input-output analysis, model reduction and control of the flat-plate boundary layer," Journal of Fluid Mechanics, Vol. 620, 2009, pp. 263-298.

${ }^{17}$ Bagheri, S., Henningson, D., Hoepffner, J., and Schmid, P., "Input-output analysis and control design applied to a linear model of spatially developing flows," Applied Mechanics Reviews, Vol. 62, No. 2, 2009, pp. 020803.

${ }^{18}$ Semeraro, O., Pralits, J. O., Rowley, C. W., and Henningson, D. S., "Riccati-less approach for optimal control and estimation: an application to two-dimensional boundary layers," Journal of Fluid Mechanics, Vol. 731, 2013, pp. $394-417$.

${ }^{19} \mathrm{Li}$, Y. and Gaster, M., "Active control of boundary-layer instabilities," Journal of Fluid Mechanics, Vol. 550, 2006, pp. 185-205. 
${ }^{20}$ Gautier, N. and Aider, J.-L., "Feed-forward control of a perturbed backward-facing step flow," Journal of Fluid Mechanics, Vol. 759, 2014, pp. 181-196.

${ }^{21}$ Devasia, S., "Should model-based inverse inputs be used as feedforward under plant uncertainty?" IEEE Transactions on Automatic Control, Vol. 47, No. 11, 2002, pp. 1865-1871.

${ }^{22}$ Press, W. H., Numerical recipes 3rd edition: The art of scientific computing, Cambridge university press, 2007.

${ }^{23}$ Hahn, S. L., Hilbert transforms in signal processing, Vol. 2, Artech House Boston, 1996.

${ }^{24}$ Cheung, L. C. and Lele, S. K., "Linear and nonlinear processes in two-dimensional mixing layer dynamics and sound radiation," Journal of Fluid Mechanics, Vol. 625, 2009, pp. 321-351.

${ }^{25}$ Colonius, T., Lele, S. K., and Moin, P., "Sound generation in a mixing layer," Journal of Fluid Mechanics, Vol. 330, 1997, pp. 375-409.

${ }^{26}$ Wei, M. and Freund, J., "A noise controlled free shear flow," Journal of Fluid Mechanics, Vol. 546, 2006, pp. $123-152$.

${ }^{27}$ Mitchell, B. E., Lele, S. K., and Moin, P., "Direct computation of the sound generated by vortex pairing in an axisymmetric jet," Journal of Fluid Mechanics, Vol. 383, 1999, pp. 113-142.

${ }^{28}$ Violato, D. and Scarano, F., "Three-dimensional vortex analysis and aeroacoustic source characterization of jet core breakdown," Physics of Fluids (1994-present), Vol. 25, No. 1, 2013, pp. 015112. 